\title{
Terapia secuencial: una opción en el paciente hemofílico que no responde a la terapia puente
}

\author{
- Claudia Sossa, María Helena Solano, Claudia Patricia Casas, Ángela Peña
}

Clínica Foscal, UNAB, Hospital San José, FUCS, Bogotá, D.C.

Contacto: claudiapatriciacasas@gmail.com

Introducción y objetivos. El tratamiento para hemofilia severa con inhibidores de alta respuesta disponible es APC y aFVII. Los pacientes pueden fallar a esta terapia. Reporte de cinco casos de pacientes con hemofilia severa con inhibidores, con eventos de sangrado mayor, que no logran mejoría con monoterapia y requieren manejo secuencial con dos productos de terapia puente.

Materiales y métodos. Reporte de casos, las variables cualitativas se presentan con frecuencias absolutas y relativas. Las variables cuantitativas se resumen con medidas de tendencia central.

Resultados. Cinco pacientes hemofílicos con inhibidores de alta respuesta con eventos de sangrado. La mediana de edad: 20 años, mediana de días de monoterapia: 10, días de terapia secuencial: 8,6, tiempo para resolver el sangrado: 4 . No eventos trombóticos.

Conclusiones. La hemofilia con inhibidores de alta respuesta es una condición de muy baja frecuencia. La terapia secuencial es una opción para pacientes con inhibidores que no responden a la monoterapia puente y que requieren control hemostático.

\section{Hemofilia A adquirida asociada a lupus eritematoso sistémico y hepatitis autoinmune. Reporte de un caso y revisión de la literatura}

\author{
- José Domingo Saavedra Ramírez, Julián Esteban Londoño Hernández
}

Fundación Colombiana de Cancerología, Clínica Vida, Medellín (Antioquia).

Contacto: jsaavedra@une.net.co

Introducción y objetivos. La hemofilia A adquirida es un trastorno de la coagulación caracterizado por una alteración en los niveles del factor VIII secundaria a la producción de autoanticuerpos neutralizantes y está asociada a enfermedades autoinmunes. Nuestro objetivo es presentar el caso clínico de una paciente con antecedente de hepatitis autoinmune y lupus eritematoso sistémico, quien desarrolló hemofilia A adquirida. Describimos su evolución, respuesta terapéutica y aportamos una revisión actualizada sobre el tema.

Materiales y métodos. Se realiza toma de consentimiento informado a la paciente, donde se declara explícitamente la protección de la información y aceptación del uso de esta, acorde a lo promulgado en las buenas prácticas clínicas.
Resultados. La paciente fue tratada con factor VII activado recombinante e inmunosupresores. En poco tiempo, se logró la disminución del inhibidor y normalización de la coagulación.

Conclusiones. Este caso es el número 19 reportado en la literatura y orienta el manejo actual de esta condición.

\section{Tratamiento de síndrome antifosfolípidos con células mesenquimatosas}

\section{- Carlos Enrique Sanabria Labrador}

\section{Contacto: csanalab@gmail.com}

Introducción y objetivos. El síndrome antifosfolípidos es un estado autoinmune de hipercoagulabilidad causado por anticuerpos dirigidos contra los fosfolípidos de las membranas celulares. Hace más de una década se están investigando las propiedades y los efectos sobre el sistema inmunológico de las células mesenquimatosas, encontrándose que no expresan proteínas para grupo sanguíneo solo HLA tipo I. El objetivo del trabajo es evaluar la efectividad de las células mesenquimatosas heterólogas para frenar el síndrome antifosfolípidos en cinco pacientes de sexo femenino con categoría I según criterios de consenso 2006.
Materiales y métodos. Se toman muestras de médula del paciente y de dos familiares, se someten a cultivos e hibridación; a las tres semanas se aplican células mesenquimatosas intravenosas.

Resultados. Se realiza evaluación durante los tres años siguientes, documentándose normalización de los títulos de anticardiolipinas y anticoagulante lúpico; todas las pacientes permanecieron asintomáticas.

Conclusiones. Existe la posibilidad de tratamiento con células mesenquimatosas heterólogas para pacientes con síndrome antifosfolípidos. 